\title{
Degradation behaviors of nonylphenol ethoxylates by isolated bacteria using improved isolation method
}

\author{
GU Xin ${ }^{1}$, ZHANG Yu ${ }^{1, *}$, ZHANG Jing ${ }^{1}$, YANG Min ${ }^{1, *}$, Hideyuki Tamaki², Yoichi Kamagata ${ }^{2}$. \\ 1. State Key Laboratory of Environmental Aquatic Chemistry, Research Center for Eco-Environmental Sciences, \\ Chinese Academy of Sciences, Beijing 100085, China. E-mail: bpguxin@gmail.com \\ 2. Institute for Biological Resources and Functions, National Institute of Advanced Industrial Science and Technology \\ Tsukuba, Ibaraki 305-8566, Japan
}

Received 21 May 2008; revised 2 July 2008; accepted 23 July 2008

\begin{abstract}
Nonylphenol ethoxylate (NPEO)-degrading bacteria were isolated from activated sludge using an improved isolation method, and the corresponding degradation behaviours were investigated. Eight NPEO-degrading strains distributed in genera Pseudomonas, Sphingomonas, Sphingobium, Cupriavidus, Ralstonia, Achromobacter, and Staphylococcus were acquired. The latter five genera have never been reported for the degradation of NPEOs. Four degradation patterns were observed for the eight pure strains. In pattern A, NPEOs were converted to short-chain NPEOs and carboxylated products, while in pattern B, lower ethoxylated oligomers appeared. Nonylphenol monoethoxylate was the main product in pattern $\mathrm{C}$, while in pattern D ethoxylated units was oxidized but not shortened. Pattern C and D have not yet been reported.
\end{abstract}

Key words: nonylphenol ethoxylate (NPEO); biodegradation; ssolation method

Nonylphenol ethoxylates (NPEOs) are a major class of nonionic surfactants used in large amounts in industrial and agriculture applications (Thiele et al., 1997). The environmental fate of NPEOs has received wide attention because some of their biodegradation intermediates in different aquatic environments, such as nonylphenol (NP), nonylphenol monoethoxylate $\left(\mathrm{NP}_{1} \mathrm{EO}\right)$, nonylphenol diethoxylate $\left(\mathrm{NP}_{2} \mathrm{EO}\right)$ and low ethoxylated nonylphenoxy carboxylates (NPECs) act as estrogenic hormone to aquatic organisms, mammals and birds at low concentration (Jobling and Sumpter, 1993; Jobling et al., 1996; White et al., 1994).

NPEOs enter the environment primarily via industrial and municipal wastewater treatment plants (WWTPs) (Ying et al., 2002), where higher ethoxylated NPEOs are efficiently bioconverted into low ethoxylated products. Thus, the biodegradation process converts the relatively unharmful surfactants into more endocrine-disruptive components. An understanding of this bioconversion mechanism by isolated strains will be important to assess environmental risk for this group of products. Until now, most of the isolated bacteria showing the ability to assimilate NPEOs belong to the class $\gamma$-Proteobacteria (mainly the genus Pseudomonas, Acinetobacter, Aeromonas). However, culture independent (molecular) studies on NPEO-degrading activated sludge reactors (Lozada et

\footnotetext{
* Corresponding author. E-mail: yangmin@rcees.ac.cn (Yang Min); zhangyu@ @rcees.ac.cn.
}

al., 2004, 2006) and natural water microcosms (Zhang et al., 2008a) indicated that the $\alpha$-Proteobacteria and $\beta$-Proteobacteria play an important role in NPEO degradation. It has been reported that some improved cultivationbased technique permitted successful isolation of unknown or novel bacteria (Tamaki et al., 2005). Therefore, the object of this work was to isolate novel NPEO-degrading bacteria with high genetic diversity from activated sludge by using improved cultivation-based technique and thereafter explore the corresponding degradation behaviors.

The bacterial strains in this study were isolated from activated sludge acclimated with NPEOs surfactant mixtures (technical grade, with an ethoxylate units (EOs) range of 4-19 and average EO chain length of 9, $\mathrm{NP}_{\mathrm{av} 9} \mathrm{EO}$ ) in a simulated NPEO-degrading bioreactor (Zhang et al., 2008). Eight NPEO-degrading bacteria were isolated using an improved isolation method (Tamaki et al., 2005) with a PE03 medium containing $50 \mathrm{mg} / \mathrm{L} \mathrm{NP}_{\mathrm{av} 9} \mathrm{EO}$ as the sole added carbon source. The isolated NPEOdegrading bacteria belong to $\alpha-, \beta-, \gamma$-Proteobacteria and Firmicutes Phylum (Fig.1), and the members of $\beta$ - and $\alpha$ Proteobacteria were dominated, which is consistent with the results obtained by culture independent methods from an $\mathrm{NP}_{10} \mathrm{EO}$-amended sludge reactor (Lozada et al. 2004, 2006).

The 8 isolates were assigned to seven different genera: Pseudomonas (NP15b), Sphingomonas (NP41b, NP42a), Sphingobium (NP22b), Cupriavidus (NP213b), Ralstonia 


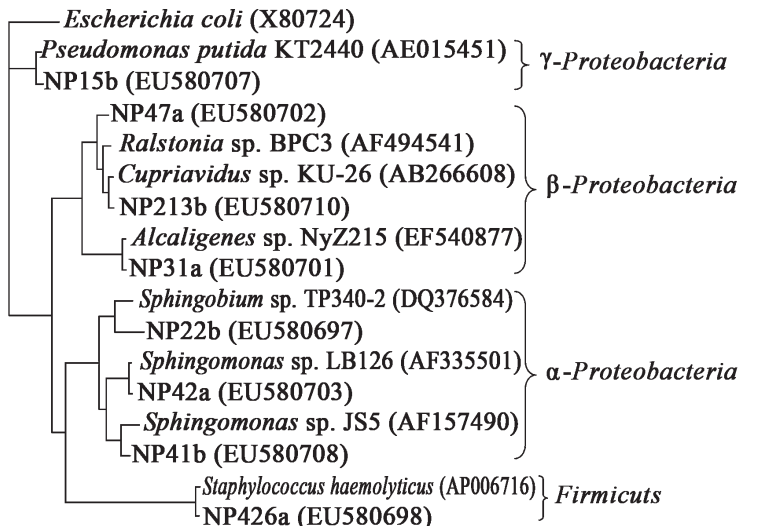

Fig. 1 Phylogenetic distribution of NPEOs-utilizing cultures isolated from enrichment cultures. Calculation of the phylogenetic tree was based on the neighbor-joining method with bootstrapping. The tree was rooted with the 16S rRNA gene sequence of Escherichia coli as the outgroup.

(NP47a), Achromobacter (NP31a), and Staphylococcus (NP426a) (Fig.1). To our knowledge, the latter five genera were the first time to be reported for the degradation of NPEOs.

The growth and degradation tests were performed for each isolates in liquid PE03 medium with $500 \mathrm{mg}$ of $\mathrm{NP}_{\mathrm{av} 9} \mathrm{EO}$ per liter as the sole carbon source. Seven isolates could degrade $\mathrm{NP}_{\mathrm{av} 9} \mathrm{EO}$ within $2 \mathrm{~d}$, while strain Ralstonia NP47a showed a poor but visible growth and a partial degradation. No abiotic NPEO removal was observed (data not shown).

$\mathrm{NP}_{\mathrm{av} 9}$ EO biodegradation intermediates were analyzed by liquid chromatography/mass spectrometry (LC/MS) and gas chromatography/mass spectrometry (GC/MS). NPEO degradation could be classified into four patterns (patterns A-D) based on different degradation products.

In pattern $\mathrm{A}$, long homologs $(n>4)$ disappeared within $2 \mathrm{~d}$, while more persistent intermediate homologs such as $\mathrm{NP}_{2} \mathrm{EO}$ and $\mathrm{NP}_{3} \mathrm{EO}$ and short-chain NPECs accumulated as the major end products. Four strains (Sphingobium NP22b, Sphingomonas NP41b, Cupriavidus NP213b, Staphylococcus NP426a) showed this degradation pattern, and typical changes of the four short-chain NPEOs and NPECs by NP22b are shown in Fig.2. These compounds are the most frequently reported intermediates formed during the aerobic biodegradation of NPEOs (Jonkers et al., 2001; Hayashi et al., 2005).

The concentrations of the carboxylated products were relatively low in the first two days, and the concentrations of NPnEC $(n>4)$ remained low during the tested period. After the formation of short chain oligomers reached a plateau, the concentrations $\mathrm{NP}_{2} \mathrm{EC}$ and $\mathrm{NP}_{3} \mathrm{EC}$ increased rapidly, with $\mathrm{NP}_{2} \mathrm{EC}$ reaching a maximum concentration of $102 \mu \mathrm{mol} / \mathrm{L}$ ( $47 \%$ of the total intermediates molar concentration).

Most NPEO-degrading strains reported in previous publications can be classified into pattern $\mathrm{A}$, and most of reported strains belong to genera Pseudomonas (Maki et al., 1994; Sato et al., 2001) of $\gamma$-Proteobacteria. Our study indicated that the pattern A NPEO-degrading microbes existed diversely, belonging to $\alpha$-Proteobacteria, $\beta$-Proteobacteria, and Firmicuts.

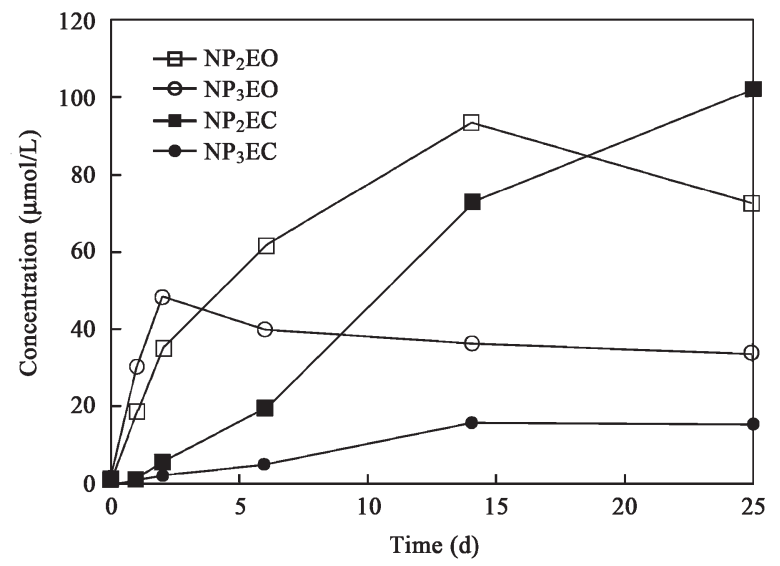

Fig. 2 Changes in the concentrations of typical metabolites during the biodegradation period by strain NP22b (Pattern A).

In pattern B (Achromobacter NP31a and Pseudomonas $\mathrm{NP} 15 \mathrm{~b}$ ), except for $\mathrm{NP}_{2} \mathrm{EO}$ and $\mathrm{NP}_{2} \mathrm{EC}$ as the main degradation products, two new metabolites, $\mathrm{NP}_{1} \mathrm{EO}$ and its carboxylated product $\mathrm{NP}_{1} \mathrm{EC}$ were produced within $14 \mathrm{~d}$ and accumulated gradually (data not shown). The concentration of $\mathrm{NP}_{1} \mathrm{EO}$ increased to around $25 \mu \mathrm{mol} / \mathrm{L}$ in $25 \mathrm{~d}$, which is nearly $30 \%$ of the concentration of $\mathrm{NP}_{2} \mathrm{EO}$. Growth reached the maximum in $1 \mathrm{~d}$, and complete transformation of $\mathrm{NP}_{\mathrm{av} 9} \mathrm{EO}$ occurred within $1 \mathrm{~d}$ together with the maximum growth of microbes. It has been reported that Sphingomonas macrogoltabidus G-3 isolated from topsoil of golf courses is capable of bioconverting octylphenol ethoxylates (OPEOs) to small amount of octylphenol monoethoxylate $\left(\mathrm{OP}_{1} \mathrm{EO}\right)$ together with large amount of $\mathrm{OP}_{2} \mathrm{EO}$ (Nishio et al., 2005).

In pattern $\mathrm{C}$ (Sphingomonas $\mathrm{NP} 42 \mathrm{a}$ ), $\mathrm{NP}_{1} \mathrm{EO}$ was the main degradation products (Fig.3), and the concentrations of the carboxylated products was very low $(<4 \%)$. $\mathrm{NP}_{1} \mathrm{EO}$ is known as the most toxic and estrogenic in NPEOs homologue. $\mathrm{NP}_{1} \mathrm{EO}$ was identified as a main metabolite from aerobic and anaerobic degradation of NPEOs in many of the bench-scale studies as well as in field studies, nevertheless there is little information about the bioconversion mechanisms and the pure strains which are capable to

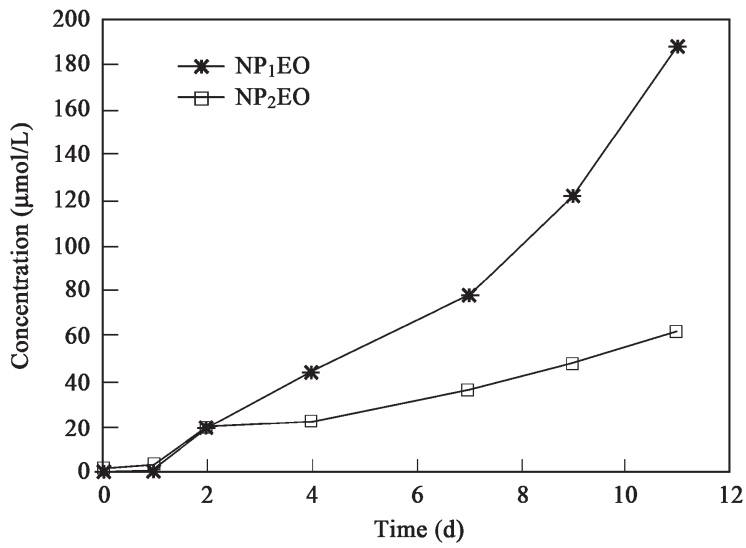

Fig. 3 Changes in the concentrations of $\mathrm{NP}_{1} \mathrm{EO}$ and $\mathrm{NP}_{2} \mathrm{EO}$ during the biodegradation period by Strain NP42a (Pattern C). 
convert NPEOs to $\mathrm{NP}_{1} \mathrm{EO}$. This is the first report about $\mathrm{NP}_{1} \mathrm{EO}$ producing pure culture, which will improve the understanding of bioconversion mechanism of NPEOs.

In pattern D (Ralstonia NP47a), NPEOs were converted to their carboxylated products without the formation of lower ethoxylated oligomers (Fig.4). Until now almost all studies on NPEO biodegradation by pure culture indicated that the degradation proceeds via the successive exoscission of the ethylene oxide chain (Maki et al., 1994; Jones et al., 1998; Sato et al., 2001). It is very interesting that strain NP47a has the ability to oxidize the EO chain, accordingly lacks the ability to shorten the EO chain. The only NPEOs biodegradation mechanism that has been validated by axenic strains is the EO chains exoscission pathways (oxidative and non-oxidative). However, pattern D exhibits many differences from the reported pathways, and the further mechanism is still under study.
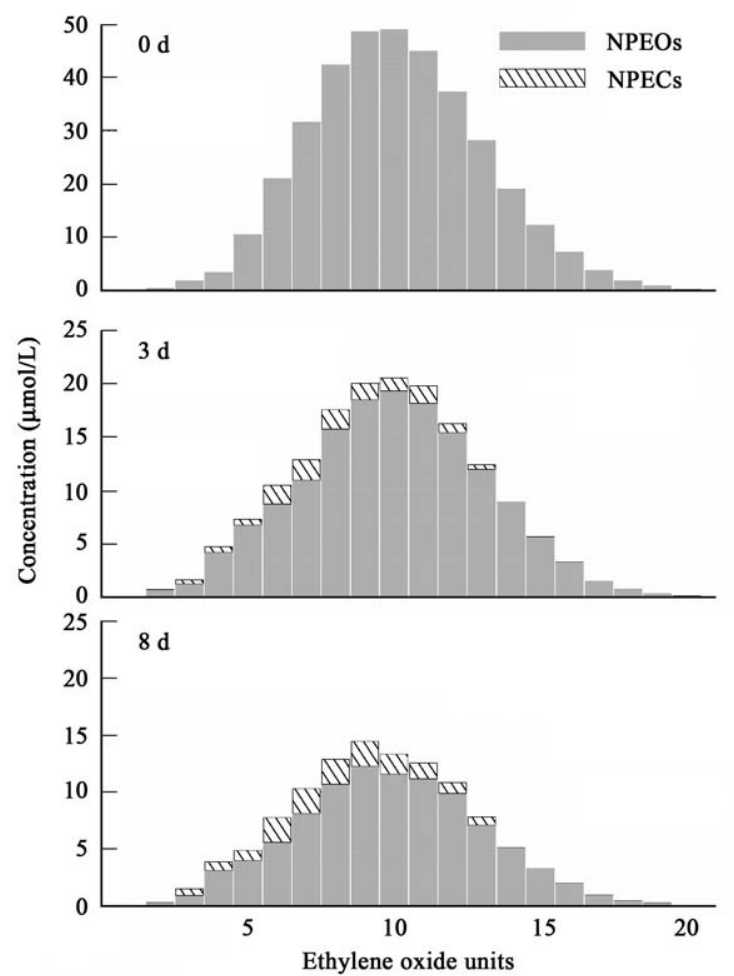

Fig. 4 Time course of $\mathrm{NP}_{\mathrm{av} 9} \mathrm{EO}$ degradation by strain NP47a (Pattern D).

As a conclusion, eight NPEO-degrading bacteria belong to $\alpha-, \beta-, \gamma$-Proteobacteria and Firmicutes Phylum and 7 different genera were successfully isolated from the NPEO degrading bioreactor by an improved isolation method. Five of the 7 genera have not yet been reported previously for the biodegradation of NPEOs. These 8 isolates demonstrated four different degradation patterns, and two of them have not yet been reported. This study improved the understanding of bioconversion process of NPEOs in the environment.

\section{Acknowledgements}

This work was supported by the National Natural Science Foundation of China (No. 50578153, 50525824) and the Ministry of Science and Technology, China (No. 2006DFA91870).

\section{References}

Hayashi S, Saito S, Kim J H, Nishimura O, Sudo R, 2005. Aerobic biodegradation behavior of nonylphenol polyethoxylates and their metabolites in the presence of organic matter. Envirol Sci Technol, 39(15): 5626-5633.

Jobling S, Sheahan D, Osborne J A, Matthiessen P, Sumpter J P, 1996. Inhibition of testicular growth in rainbow trout (oncorhynchus mykiss) exposed to estrogenic alkylphenolic chemicals. Environ Toxicol Chem, 15(2): 194-202.

Jobling S, Sumpter J P, 1993. Detergent components in sewage effluent are weakly oestrogenic to fish: An in vitro study using rainbow trout (Oncorhynchus mykiss) hepatocytes. Aqua Toxicol, 27(3-4): 361-372.

Jones F W, Westmoreland D J, 1998. Degradation of nonylphenol ethoxylates during the composting of sludges from wool scour effluents. Environ Sci Technol, 32(17): 2623-2627.

Jonkers N, Knepper T P, De Voogt P, 2001. Aerobic biodegradation studies of nonylphenol ethoxylates in river water using liquid chromatography-electrospray tandem mass spectrometry. Environ Sci Technol, 35(2): 335-340.

Lozada M, Figuerola E L M, Itria R F, Erijman L, 2006. Replicability of dominant bacterial populations after long-term surfactantenrichment in lab-scale activated sludge. Environ Microbio, 8(4): 625-638.

Lozada M, Itria R F, Figuerola E L M, Babay P A, Gettar R T, de Tullio L A, Erijman L, 2004. Bacterial community shifts in nonylphenol polyethoxylates-enriched activated sludge. Water Res, 38(8): 20772086.

Maki H, Masuda N, Fujiwara Y, Ike M, Fujita M, 1994. Degradation of alkylphenol ethoxylates by Pseudomonas sp. strain tr01. Appl Environ Microbio, 60(7): 2265-2271.

Nishio E, Yoshikawa H, Wakayama M, Tamura H, Morita S, Tomita Y, 2005. Isolation and identification of Sphingomonas sp. that yields tert-octylphenol monoethoxylate under aerobic conditions. Biosci, Biotechnol, Biochem, 69(7): 1226-1231.

Sato H, Shibata A, Wang Y, Yoshikawa H, Tamura H, 2001. Characterization of biodegradation intermediates of non-ionic surfactants by matrix-assisted laser desorption/ionization-mass spectrometry 1. bacterial biodegradation of octylphenol polyethoxylate under aerobic conditions. Polym Degrad Stab, 74(1): 69-75.

Tamaki H, Sekiguchi Y, Hanada S, Nakamura K, Nomura N, Matsumura M, Kamagata Y, 2005. Comparative analysis of bacterial diversity in freshwater sediment of a shallow eutrophic lake by molecular and improved cultivation-based techniques. Appl Environ Microbio, 71(4): 2162-2169.

Thiele B, Günther K, Schwuger M J, 1997. Alkylphenol ethoxylates: Trace analysis and environmental behavior. Chem Rev, 97(8): 3247-3272.

White R, Jobling S, Hoare S, Sumpter J, Parker M, 1994. Environmentally persistent alkylphenolic compounds are estrogenic. Endocrinology, 135(1): 175-182.

Ying G G, Williams B, Kookana R, 2002. Environmental fate of alkylphenols and alkylphenol ethoxylates: a review. Environment International, 28(3): 215-226.

Zhang J, Yang M, Zhang Y, Chen M X, 2008. Biotransformation of nonylphenol ethoxylates during sewage treatment under anaerobic and aerobic conditions. Journal of Environmental Sciences, 20(2): 135-141.

Zhang Y, Sei K, Toyama T, Ike M, Zhang J, Yang M, Kamagata Y, 2008a. Changes of catabolic genes and microbial community structures during biodegradation of nonylphenol ethoxylates and nonylphenol in natural water microcosms. Biochem Eng J, 39(2): 288-296. 